\title{
Unraveling the Solid-Liquid-Vapor Phase Transition Dynamics at the Atomic Level with Ultrafast X-Ray Absorption Near-Edge Spectroscopy
}

\author{
F. Dorchies, ${ }^{1, *}$ A. Lévy, ${ }^{1,2}$ C. Goyon, ${ }^{1}$ P. Combis,${ }^{3}$ D. Descamps, ${ }^{1}$ C. Fourment, ${ }^{1}$ M. Harmand,${ }^{1}$ S. Hulin,${ }^{1}$ \\ P. M. Leguay, ${ }^{1}$ S. Petit, ${ }^{1}$ O. Peyrusse, ${ }^{1}$ and J. J. Santos ${ }^{1}$ \\ ${ }^{1}$ Université de Bordeaux-CNRS-CEA, Centre Lasers Intenses et Applications (CELIA), Talence, F-33405, France \\ ${ }^{2}$ LULI, Ecole Polytechnique-CNRS-CEA-UPMC, route de Saclay, 91128 Palaiseau, France \\ ${ }^{3} C E A, D A M$, DIF, F-91297 Arpajon, France
}

(Received 16 June 2011; published 8 December 2011)

\begin{abstract}
X-ray absorption near-edge spectroscopy (XANES) is a powerful probe of electronic and atomic structures in various media, ranging from molecules to condensed matter. We show how ultrafast time resolution opens new possibilities to investigate highly nonequilibrium states of matter including phase transitions. Based on a tabletop laser-plasma ultrafast $\mathrm{x}$-ray source, we have performed a time-resolved $(\sim 3 \mathrm{ps})$ XANES experiment that reveals the evolution of an aluminum foil at the atomic level, when undergoing ultrafast laser heating and ablation. X-ray absorption spectra highlight an ultrafast transition from the crystalline solid to the disordered liquid followed by a progressive transition of the delocalized valence electronic structure (metal) down to localized atomic orbitals (nonmetal-vapor), as the average distance between atoms increases.
\end{abstract}

DOI: 10.1103/PhysRevLett.107.245006

PACS numbers: 52.38.Ph, 52.50.Jm, 52.70.La

The rapid development of tabletop femtosecond lasers has opened a wide range of new applications in industry and medicine. When focused onto a sample, a femtosecond laser pulse can rapidly deposit energy in a micron-size spot, driving a well-controlled matter removal (for micromachining) that can also be used for clean deposition of thin films on another surface. During the laser ablation, the matter undergoes ultrafast phase transitions whose understanding, as well as the determination of the time scale in which they occur, is primordial for the optimization of processes. From a more general point of view, femtosecond lasers offer a unique opportunity to study these phase transitions on their natural ultrafast time scales that are not reachable in the everyday experience.

Under strong pulse excitation, it is also possible to reach transient extreme states of matter with pressures exceeding the $1 \mathrm{Mbar}$ level that are not accessible in a static way. The investigation of such warm dense matter (WDM) is one of the great challenges of contemporary physics [1]. Femtosecond lasers can rapidly heat matter, leading to ultrafast solid-liquid-WDM transitions, followed by a more complex multiphase expansion at a picosecond time scale. Highly nonequilibrium states of matter are expected, due to the finite rate of energy transfer from the excited electrons to the lattice. As the atomic structure modification is supposed to be driven by the photoexcited electrons, it is of primary importance to determine the respective time scales of the evolution of both electron and atomic structures. In order to shed some light on these transient processes, the experimental challenge is then to combine such atomic-level measurements with ultrafast time resolution.

Recently, new techniques of ultrafast electron [2,3] and $\mathrm{x}$-ray diffraction [4,5] have been developed [6]. Although they have been successfully used to investigate coherent phonon dynamics [7], melting phenomena [8,9], or electronic bond hardening in solid-WDM transition [10], they are not adapted where no long-range order is expected (liquid, WDM, vapor). Ultrafast x-ray absorption nearedge spectroscopy (XANES) is particularly suited for such situations [11] since it probes the short-range order that can persist well beyond the melting. Also, it basically probes the unoccupied electron density of states (DOS), giving access to the dynamic interplay between the valence electrons and the atomic arrangement.

In contrast to ultrafast diffraction that has been first demonstrated with versatile and accessible tabletop laser systems, the development of tabletop ultrafast x-ray absorption experiments has been limited, due to the very low $\mathrm{x}$-ray fluxes reported in continuous spectra from bremsstrahlung emission [12,13]. The first demonstration of a few picosecond resolution has been reported on resonant absorption line vanishing during molecule photodissociation [14]. First ultrafast XANES experiments have just been recently reported on synchrotronlike facilities: coupling a few ps resolution streak camera with a synchrotron [15], or using the slicing scheme to reach subpicosecond time resolution $[16,17]$. But in both cases, the very low number of x-ray photons available per shot severely limits their use for large excitation levels, well above damage threshold, where the sample must be refreshed before each laser shot.

In order to overcome these limitations, we have developed a bright tabletop $\mathrm{x}$-ray source suitable for XANES experiments [18], by optimizing the broad $M$-band thermal emission from laser-produced plasmas in the multi-keV range [19]. The number of photons is increased by orders 
of magnitude compared with the bremsstrahlung emission previously considered $[12,13]$. The isotropic nature of the pointlike $\mathrm{x}$-ray source allows the use of an energydispersive $\mathrm{x}$-ray absorption device, providing a full spectrum for each laser shot. As a consequence, a few tens of shots are just needed to get exploitable XANES spectra as presented in this Letter, using $100 \mathrm{~mJ}$ laser pulses operated at $10 \mathrm{~Hz}$ repetition rate. This is an important step forward in ultrafast x-ray absorption fine spectroscopy.

We have used this tabletop $\mathrm{x}$-ray source to perform the time-resolved XANES investigation of a femtosecond laser-ablated aluminum sample, near the Al $K$ edge. A sketch of the experimental setup is reported in Fig. 1. The laser beam is separated in two synchronized pulses. The $1000 \AA$ thick aluminum sample is heated with the first laser pulse (120 fs) on a $700 \mu \mathrm{m} \times 350 \mu \mathrm{m}$ ellipsoidal zone. Its fluence is set to $6 \mathrm{~J} / \mathrm{cm}^{2}$, more than 1 order of magnitude higher than the ablation threshold [20], in order to avoid the possible nucleation (liquid-vapor coexistence regime) in the first tens of picosecond of the expansion [21]. The second laser pulse is focused on the $\mathrm{x}$-ray conversion target

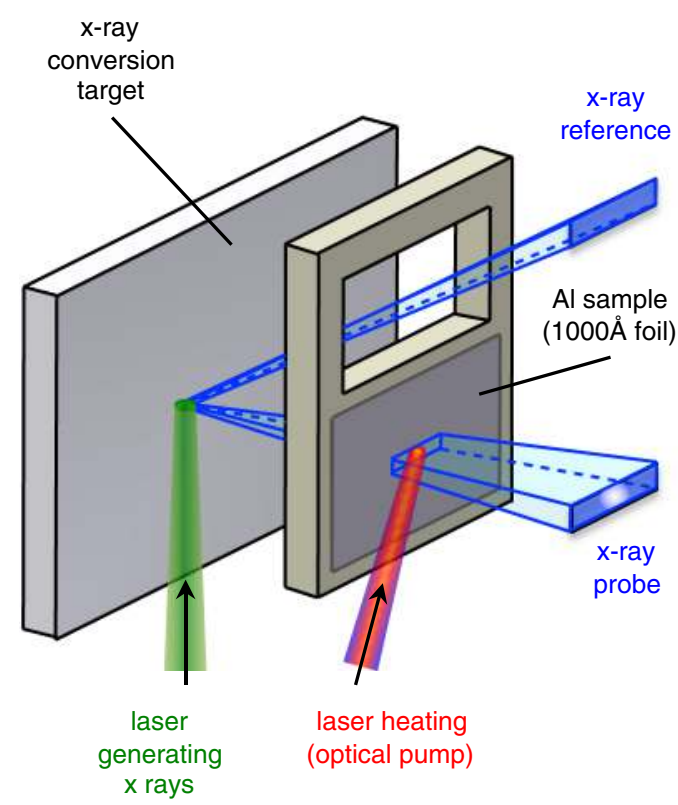

FIG. 1 (color online). Schematic of the time-resolved x-ray absorption setup. The first laser pulse (optical pump) heats the $1000 \AA$ thick aluminum sample that is deposited on a specific grid. The second laser pulse is focused on the $\mathrm{x}$-ray conversion target that is set $7 \mathrm{~mm}$ behind the sample, and generates a bright, broadband, isotropic, and ultrafast $\mathrm{X}$-ray source. $\mathrm{X}$ rays are spectrally dispersed by two different Bragg crystals (not represented) on a CCD camera: the upper one directly measures the $\mathrm{x}$-ray source spectrum (x-ray reference), while the lower one measures the transmitted spectrum through the heated aluminum sample (x-ray probe). The solid angles of respective collected $\mathrm{x}$-ray emission are represented. The delay between laser pulses is varied in order to obtain time-resolved XANES with respect to the heating. (holmium) on a $80 \mu \mathrm{m}$ diameter spot to produce the ultrafast isotropic $\mathrm{x}$-ray emission. This emission is collected and dispersed by two Bragg crystals on a CCD camera (chargecoupled device), respectively, recording the reference spectrum of the X-ray source and the transmitted spectrum through the laser-heated sample. Details about the spectrometer and the procedure for XANES spectra extraction have been previously reported in [22]. The device provides a spatial resolution of $\sim 100 \mu \mathrm{m}$ along the horizontal axis in Fig. 1, allowing a careful alignment between the sample heated area and the probed one. The time between optical pump and x-ray probe is monitored using a delay line, providing a jitter-free adjustable delay. The time resolution is mainly limited by the $\mathrm{x}$-ray pulse duration of $3.15 \pm 0.25 \mathrm{ps} r \mathrm{rm}$, which has been measured with a streak camera.

The time-resolved XANES spectra are reported in Fig. 2. Each one is obtained after integration of the $\mathrm{x}$ rays that are collected through a $450 \mu \mathrm{m} \times 140 \mu \mathrm{m}$ rectangular zone of the sample, in which the heating flux is homogeneous within $20 \%$. The first spectrum, recorded 7.5 ps before the optical pump pulse, is identical to a cold solid aluminum spectrum (i.e., measured without heating). It shows a sharp $K$ edge that reveals the clear separation between occupied and unoccupied states in the conduction band at the Fermi energy. The $K$ edge is followed by XANES modulations

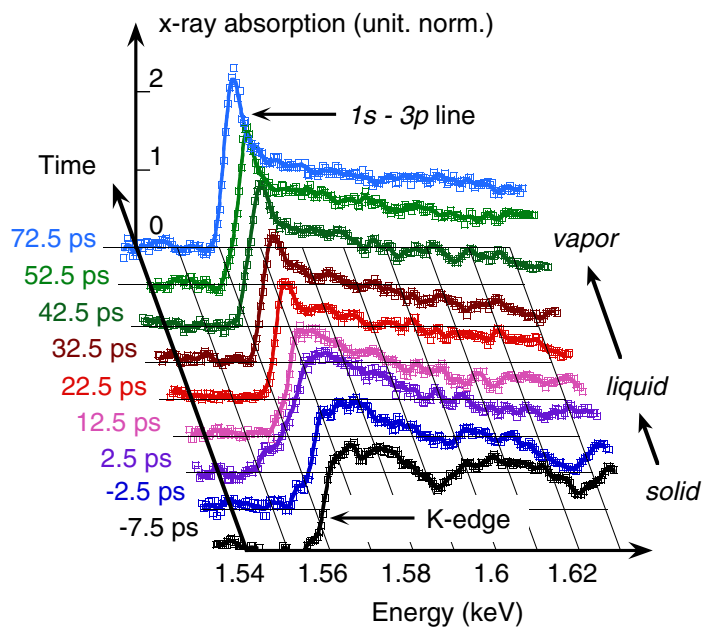

FIG. 2 (color online). XANES spectra measured as a function of the time delay between the X-ray probe and the optical pump. The origin is set when the maxima of optical and x-ray pulses coincide. The spectrum registered at the delay -7.5 ps exhibits the same features as for unheated Al sample: a sharp $K$ edge at $1.559 \mathrm{keV}$, followed by XANES modulations. These modulations vanish promptly after heating, revealing a fast loss of atomic short-range order (therefore an ultrafast solid-liquid transition). At delay $2.5 \mathrm{ps}$, the $K$-edge slope is broadened due to a high electron temperature $T_{e}$. At longer delays, a transition from $K$ edge to $1 s-3 p$ atomic absorption line can be observed, revealing the progressive relocalization of valence electrons in atomic orbitals during the liquid-vapor transition. 
that are expected from the face-centered cubic (fcc) lattice structure of solid aluminum. These modulations completely disappear $2.5 \mathrm{ps}$ after the heating pulse, indicating a rapid loss of the short-range order and evidencing a fast solid-liquid transition. The same spectrum also exhibits a broadening of the $K$-edge slope that reveals the expected thermal broadening of the Fermi level. A Fermi-Dirac fit [23] indicates an electronic temperature of $1.8 \pm 0.5 \mathrm{eV}$. At longer delays $(\sim 20 \mathrm{ps})$, a progressive transition is observed from the absorption $K$ edge to the $1 s-3 p$ atomic absorption line.

The macroscopic evolution of the heated sample has been estimated with a two-temperature hydrodynamic code [20]. As the sample thickness is slightly larger than the laser skin depth, significant longitudinal gradients of temperature are initially present, then smoothed by the thermal conduction after a few picoseconds. The values of electron temperature $T_{e}$, ion temperature $T_{i}$ and density $N_{i}$, averaged over the sample thickness, are reported in Fig. 3. The inhomogeneities (defined as the standard deviation along longitudinal profiles) are reported in the error bars around the respective mean values that correspond to the delays of XANES measurements. The laser energy is rapidly deposited in the electrons (120 fs). Then the energy transfer starts from the electrons to the lattice. The equilibration time between electron and ion temperatures is about 10 ps. Because of the high level of initial electron heating, $T_{i}$ reaches the melting temperature $(0.08 \mathrm{eV})$ very quickly, suggesting that the solid-liquid transition occurs in

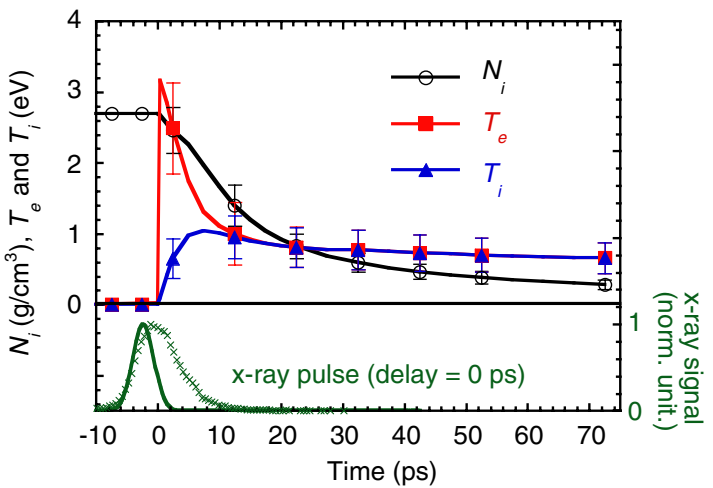

FIG. 3 (color online). Aluminum density and temperature temporal profiles as calculated with a two-temperature hydrodynamic code. The $1000 \AA$ thick aluminum is heated with $6 \mathrm{~J} / \mathrm{cm}^{2}$ laser pulse at $60^{\circ}$ incidence in $p$ polarization. Top: the density $N_{i}$, the electron temperature $T_{e}$ and the ion temperature $T_{i}$ are plotted as a function of time (solid lines). For comparison with experimental data of Fig. 2, the calculated values corresponding to the delays of XANES measurements are also indicated by symbols (circle $=N_{i}$, square $=T_{e}$, triangle $=T_{i}$ ). Bottom: the measured $\mathrm{x}$-ray temporal profile is reported (cross) for $\mathrm{x}$-ray probe optical pump delay $=0 \mathrm{ps}$, and compared to the temporal response of the streak camera (line). After deconvolution, the x-ray source duration is $3.15 \pm 0.25$ ps rms. a subpicosecond time scale. As a short-range order is still expected in the liquid phase, XANES modulations remain, and then progressively disappear when $T_{i}$ increases. Actually, $2.5 \mathrm{ps}$ after heating, XANES modulations have disappeared while estimated $T_{i}$ reaches $0.65 \pm 0.25 \mathrm{eV}$. This is consistent with calculations previously reported on isochoric heating with protons [23] where XANES modulations have been observed to vanish for temperature between 0.5 and $1 \mathrm{eV}$ at the thermal equilibrium. At the same time delay $(2.5 \mathrm{ps}), T_{e}$ is estimated to be $2.5 \pm 0.7 \mathrm{eV}$, which is consistent with the value of $1.8 \pm 0.5 \mathrm{eV}$ inferred from the measured $\mathrm{x}$-ray absorption $K$-edge broadening.

The calculation reported in Fig. 3 shows that the expansion proceeds in the same time scale as the electron ion equilibration. During the observation time reported in this study, the sample stays above the liquid-gas critical point [24], preventing from any nucleation. In these conditions, the average distance increase between neighboring atoms is directly defined by the hydrodynamic expansion. The progressive transition that we have observed in Fig. 2 from $K$ edge to the $1 s-3 p$ atomic absorption line occurs about 20 ps after heating, i.e., when the ion density $N_{i}$ reaches one third of the solid density $(\sim 1 \mathrm{~g} / \mathrm{cc})$. At this time delay, the average distance between atoms has increased by $\sim 40 \%$ from the solid case. The observed x-ray absorption spectra are in good agreement with a recent experiment and ab initio quantum molecular dynamics (QMD) simulations performed at the thermal equilibrium [25]. It was predicted that such a transition from $K$ edge to $1 s-3 p$ line should occur as soon as the density decreases below $1 \mathrm{~g} / \mathrm{cc}$.

The experiment provides the atomic-level data on the solid-liquid-vapor ultrafast transition of an aluminum sample driven by femtosecond laser-ablation, corresponding to the low temperature part of the WDM densitytemperature phase diagram (a few eV). Figure 4 summaries the deduced evolution of lattice and electron structures. In the initial solid phase, the valence electrons are delocalized in the conduction band of aluminum. They are rapidly heated by the laser pulse that broadens the Fermi occupation factor in the electron energy distribution. The electron-ion energy transfer drives an ultrafast transition from a crystalline cold solid to a disordered metal liquid, and leads to a loss of the atomic short-range order in such a warm dense state of matter. The solid-liquid transition is observed to occur faster than 2.5 ps after laser excitation. Calculations suggest that the ion temperature exceeds the melting temperature in less than 1 ps due to the large excitation level $\left(6 \mathrm{~J} / \mathrm{cm}^{2}\right)$. That transition time is significantly shorter than $3.5 \mathrm{ps}$ reported in a previous electron diffraction experiment at $70 \mathrm{~mJ} / \mathrm{cm}^{2}$ [2]. Unlike diffraction signal, which disappears when the crystalline order is broken, XANES spectra provide electron and atomic structural data beyond melting. A transition from liquid to atomic vapor is observed in a longer time scale $(\sim 20 \mathrm{ps})$, revealed by a progressive 


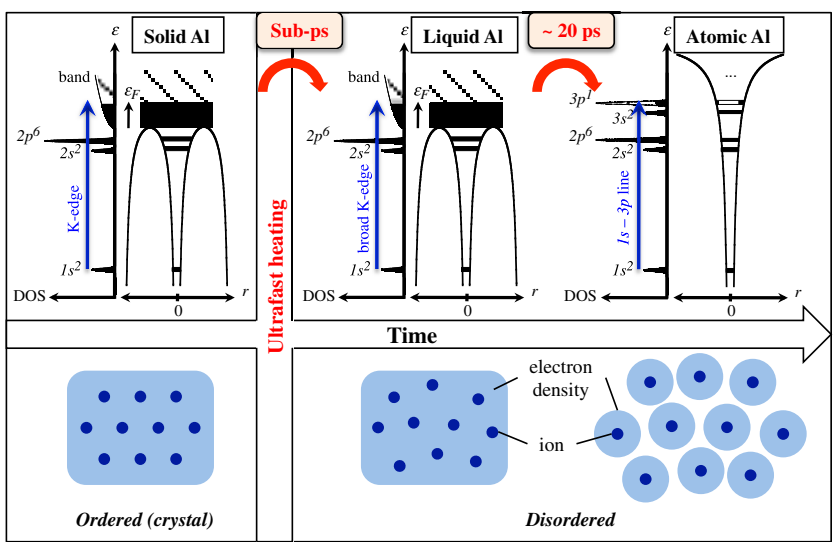

FIG. 4 (color online). Schematic of the aluminum solid-liquidvapor transition dynamics at the atomic level. Top: density of states (DOS) and localization of electrons with corresponding $\mathrm{X}$-ray absorption spectra features as measured from the $1 s$ level (vertical arrows). The potential is presented as a function of the distance $r$ from the aluminum nucleus (right side of each figure). The occupation of electron DOS (left side of each figure) is indicated in black. Bottom: Simple two-dimensional representation of the spatial distribution of ions and electron density in each phase. Solid Al: in the solid case, the conduction band is partially occupied by valence electrons up to the Fermi energy $E_{F}$, leading to a sharp x-ray absorption $K$ edge. Liquid $\mathrm{Al}$ : the laser deposits energy in the valence electrons and induces a thermal broadening of the band occupation, leading to the $K$-edge broadening. The energy is transferred from electrons to the lattice, breaking the crystalline order. Atomic Al: due to high thermal pressure, hydrodynamic expansion occurs driving the transition to atomic vapor. In isolated atoms, the electrons are localized on the atomic orbitals. As the $3 p$ orbital is partially unoccupied, the $1 s-3 p$ line is observed in the X-ray absorption spectrum.

relocalization of the valence electrons from the degenerated conduction band (metal) to the atomic orbitals (nonmetal), as the average distance between neighboring atoms increases. Calculations are coherent with experimental results, showing that this time scale is driven by the hydrodynamic expansion that depends on the thermal pressure level achieved after heating.

Besides the fundamental interest of phase transitions dynamics, ultrafast XANES experiment is achieved with a compact tabletop all-optical-laser-based setup. The time resolution is intrinsically limited by the x-ray source duration ( $3.15 \pm 0.25 \mathrm{ps} \mathrm{rms}$ ), but could be lowered down to the subpicosecond range by using cluster target for producing the $\mathrm{x}$ rays [26], or even down to the femtosecond one by considering the collimated betatron radiation generated through the interaction of a relativistic laser pulse with a gas jet [27]. Novel opportunities are also expected from hard-x-ray free electron lasers that offer femtosecond time resolution together with a high number of photons per shot [28-30]. However, although dedicated experimental areas are planned for WDM studies, the high operating cost will necessarily limit the access. Therefore, the complementary development of tabletop ultrafast $\mathrm{x}$-ray absorption devices is expected to have a high impact in the exploration of various transient physical systems: ranging from chemical reactions to phase transitions up to extreme states of matter, and highly nonequilibrium physics.

This work is supported by the French Agence Nationale de la Recherche, under Grant OEDYP No. ANR-09BLAN-0206-01, and the Conseil Régional d'Aquitaine, under Grants POLUX No. 2010-13-04-002 and COLA2 No. 2.1.3-09010502. The authors gratefully acknowledge Rodrigue Bouillaud for his technical assistance and Capucine Medina for her precious help on the laser.

*dorchies@celia.u-bordeaux1.fr

[1] National Research Council, Frontiers in High Energy Density Physics: The X-Games of Contemporary Science (National Academic Press, Washington, DC, 2003).

[2] B. J. Siwick, J. R. Dwyer, R. E. Jordan, and R. J. D. Miller, Science 302, 1382 (2003).

[3] N. Gedik, D. S. Yang, G. Logvenov, I. Bozovic, and A. H. Zewail, Science 316, 425 (2007).

[4] C. Rischel et al., Nature (London) 390, 490 (1997).

[5] A. M. Lindenberg et al., Science 308, 392 (2005).

[6] T. Pfeifer, C. Spielmann, and G. Gerber, Rep. Prog. Phys. 69, 443 (2006).

[7] K. Sokolowski-Tinten et al., Nature (London) 422, 287 (2003).

[8] C. W. Siders et al., Science 286, 1340 (1999).

[9] A. Rousse et al., Nature (London) 410, 65 (2001).

[10] R. Ernstorfer et al., Science 323, 1033 (2009).

[11] Ch. Bressler and M. Chergui, Chem. Rev. 104, 1781 (2004).

[12] T. Lee, Y. Jiang, C. G. Rose-Petruck, and F. Benesch, J. Chem. Phys. 122, 084506 (2005).

[13] J. Chen, H. Zhang, I. V. Tomov, X. Ding, and P. M. Rentzepis, Chem. Phys. Lett. 437, 50 (2007).

[14] F. Ráksi et al., J. Chem. Phys. 104, 6066 (1996).

[15] S. L. Johnson et al., Phys. Rev. Lett. 91, 157403 (2003).

[16] A. Cavalleri et al., Phys. Rev. Lett. 95, 067405 (2005).

[17] Ch. Bressler et al., Science 323, 489 (2009).

[18] F. Dorchies et al., Appl. Phys. Lett. 93, 121113 (2008).

[19] M. Harmand et al., Phys. Plasmas 16, 063301 (2009).

[20] J. P. Colombier, P. Combis, F. Bonneau, R. Le Harzic and E. Audouard, Phys. Rev. B 71, 165406 (2005).

[21] A. M. Lindenberg et al., Phys. Rev. Lett. 100, 135502 (2008).

[22] A. Lévy et al., Rev. Sci. Instrum. 81, 063107 (2010).

[23] A. Mancic et al., Phys. Rev. Lett. 104, 035002 (2010).

[24] B. Chimier, V. T. Tikhonchuk, and L. Hallo, Phys. Rev. B 75, 195124 (2007).

[25] V. Recoules and S. Mazevet, Phys. Rev. B 80, 064110 (2009); A. Lévy et al. (to be published).

[26] F. Dorchies et al., Phys. Rev. Lett. 100, 205002 (2008).

[27] A. Rousse et al., Phys. Rev. Lett. 93, 135005 (2004).

[28] B. D. Paterson and R. Abela, Phys. Chem. Chem. Phys. 12, 5647 (2010).

[29] B. Nagler et al., Nature Phys. 5, 693 (2009).

[30] S. M. Vinko et al., Phys. Rev. Lett. 104, 225001 (2010). 